\title{
LMI-based Control Performance Analysis of Networked Control System with Random Delays and Dropouts
}

\author{
Yanpeng $\mathrm{Wu}$ \\ Department of Automation \\ Northwestern Polytechnical University \\ Xi'an, P.R.China \\ wu.yanpeng123@gmail.com \\ Xinmin Wang \\ Department of Automation \\ Northwestern Polytechnical University \\ Xi'an, P.R.China \\ wxmin@nwpu.edu.cn
}

\author{
Ying $\mathrm{Wu}$ \\ Department of Automation \\ Northwestern Polytechnical University \\ Xi'an, P.R.China \\ wuyg1226@hotmail.com
}

\begin{abstract}
In this paper, the problem of control performance analysis with decay rate of networked control system (NCS) with uncertain time delays and packet dropouts is studied, and the stabilization controller is designed to deal with the network induced issues. Gridding approach is introduced to transform the time-varying NCS model into a discrete switched linear system with finite switching rules. Sufficient conditions are given for asymptotical stability and exponential stability of proposed NCS model and state feedback controller is designed via linear matrix inequality (LMI) approach. The bound of decay rate of the system is obtained by solving a LMI optimization problem. Illustrative examples are presented to demonstrate the effectiveness of the proposed method and control performance with decay rate is analyzed based on the simulation results.
\end{abstract}

Keywords-Networked control system, Time-varying sampling period, Switched system, Decay rate, Linear matrix inequality (LMI)

\section{INTRODUCTION}

Recent advances in network technology have been applied to distributed control system, whose feedback loop is closed through a real-time network, which is termed networked control system (NCS). NCS is distributed control systems in which the communication between sensor nodes, actuator nodes and controller nodes via a shared band communication network [1-3]. The benefits of NCS are low installation cost, reduced wiring, easy maintenance and diagnosis, and so on. Examples of NCS are available in manufacturing systems, aircraft systems, teleoperation of robots, etc. In spite of the great advantages that the NCS brings, network induced delays and data packet dropout may be inevitable during transmission of digital data between control devices. These factors degrade a system's performance and possibly cause system instability [4].
How to solve the network induced issues of random time delay and packet dropout to guarantee the system stability and performance is the hot research point in literature. Research on NCS has achieved a significant amount of results[5], and different models are developed for NCS to study stability criteria or stabilizing controller design. NCS with data dropout is modeled as asynchronous dynamical systems in [6]. An iterative approach is proposed to model networked control linear system with arbitrary but finite data packet dropout as switched linear systems in [7]. Optimal gain is calculated by modeling NCS as a switched system in [8].

However, these publications are only proposing the stability criteria that guarantee the system stability and related stabilizing controller design. Measures of system stability performance have not mentioned, which quantizes the effectiveness of stabilizing controller. To the best of the authors' knowledge, the problem of stability criteria with decay rate and the performance related stabilizing controller of the NCS have not been fully investigated to date. This paper will proposed a novel method to investigate stability and control design problems with performance analysis for networked control systems with random time delays and packet dropouts.

The effect of networked induced delay on closed control loop is the most serious challenge in NCS. Researchers always make some assumptions before addressing this tough issue. Time delays have been assumed to be bounded by one sampling period in [9], a constant delay in, an independent random delay [10], and a delay with known stochastic distribution governed by the Markov chain model [11]. To consider uncertain time delays naturally without assumptions, an active timevarying sampling method is proposed in this paper to make sure time delay always less than one sampling period. Linear matrix inequality (LMI) method is adopted for controller design to guarantee the stability of the closed- 
loop networked control system with uncertain time delays and packet dropouts. A "gridding" approach [12] is introduced to convert the time varying discrete system into a switched linear system. For discrete-time switched linear systems, there are many discussions on stability and stabilization [13-14] for reference. Of particular interest is the formulation of an LMI optimization problem to find the bound of the decay rate for the switched linear system. A sufficient condition is obtained for the exponential stability of the NCS and stability performance is analyzed under the decay rate.

\section{PROBLEM FORMULATION}

Consider a linear time-invariant plant described by

$$
\dot{x}(t)=A x(t)+B u(t)
$$

where $x(t) \in \mathbf{R}^{n}$ is the state vector, $u(t) \in \mathbf{R} \mathbf{p}$ is the input vector. $A$ and $B$ are constant matrices of appropriate dimensions.

To design a network controller for NCS, time delays and packet dropouts should be taken into account. If constant sampling period is adopted, time delays may be less or greater than one sampling period which will make NCS modeling more complicated. In the following, the sampling period will be set time varying to make sure time delay is less than one sampling period. In order to achieve this goal, sensor is assumed both time-driven and eventdriven. Actuator and controller are event-driven. Suppose time axis is partitioned into equidistant small intervals and the length of each interval is $l$. Define $t_{k}$ as the $k_{t h}$ updating instant of actuator, and assume that total transmission delay from sensor to actuator of the updating signal at the instant $t_{k}$ is $\tau_{k}$. Then the next sampling instant can be selected as

$$
s_{k+1}= \begin{cases}s_{k}+n l \quad t_{k} \in\left[s_{k}+(n-1) l, s_{k}+n l\right) \\ s_{k}+T_{\max } & t_{k} \geq s_{k}+T_{\max }\end{cases}
$$

where $s_{k}$ is the $k_{t h}$ sampling instant, $T_{\max }$ is the allowable maximum sampling interval we predefined $\left(T_{\max }=N l, N\right.$ is a positive integer), $n$ is a positive integer and $0<n<N$.

Fig. 1 shows the sampling and updating conditions of NCS. if the transmission time of sampled signal at time $s_{k}$ is less than $T_{\max }$, the actuator will be updated by the signal and the sensor will be driven to do the next sampling, which is called an effective sampling instant because the signal at this sampling instant is successfully transmitted from sensor to actuator, such as $s_{1}$ and $s_{2}$ marked in Fig. 1; if the signal sampled at time $s_{k}$ has not arrived before the maximal allowable updating time $s_{k}+T_{\max }$, which means the total transmission delay is out of $T_{\max }$, the signal will be discarded and the sensor will adopt time-driven mode, such as $s_{3}$ marked in Fig.1; if packet dropout happened to the sampled signal, which can be seen as a long delay packet, the time-driven mode will adopted by sensor to do the next sampling, such as $s_{6}$ marked in Fig. 1 .

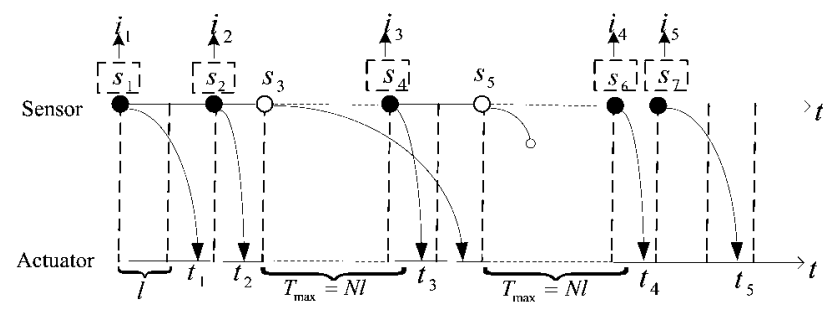

Figure 1. Sampling and Updating Conditions of NCS

Let us denote the effective sampling instant as $i_{m}$ and assume $U=\left\{i_{1}, i_{2}, i_{3}, \ldots\right\}$, which have been marked in Fig 1; correspondingly, updating instants are denoted as $S=\left\{t_{1}, t_{2}, t_{3}, \ldots\right\}$. Between two updating instants actuator operates in a zero order hold $(\mathrm{ZOH})$ fashion, meaning that the value of control signal remains constant during the interval $\left[t_{k}, t_{k-1}\right)$. Therefore, we have

$$
u(t)=u\left(i_{k}\right)=K\left(i_{k}\right) x\left(i_{k}\right), \quad t_{k} \leq t<t_{k+1}
$$

with $t_{k-1}$ being the next updating instant of the $\mathrm{ZOH}$ after tk.

Note that state-feedback control gains in (3) are not constant but varying with every updating interval $\left[t_{k}, t_{k-1}\right)$ to ensure the stability of the time varying sampled system.

Based on the selection principle of sampling instant proposed above, the time delay between sensor and actuator is ensured to be less than one sampling interval. Suppose $h_{k}$ as the length of interval between two successive effective sampling instants $i_{k}$ and $i_{k+1}$, thus, the discrete time representation of (1) can be described as follows

$$
x\left(i_{k+1}\right)=\Phi_{k} x\left(i_{k}\right)+\Gamma_{0}\left(\tau_{k}, h_{k}\right) u\left(i_{k}\right)+\Gamma_{1}\left(\tau_{k}, h_{k}\right) u\left(i_{k-1}\right)
$$

where $\Phi_{k}=e^{A h_{k}}$,

$\Gamma_{0}\left(\tau_{k}, h_{k}\right)=\int_{0}^{h_{k}-\tau_{k}} e^{A s} d s B, \Gamma_{1}\left(\tau_{k}, h_{k}\right)=\int_{h_{k}-\tau_{k}}^{h_{k}} e^{A s} d s B$.

Let us introduce a new augmented state $z(k)=\left[\begin{array}{ll}x\left(i_{k}\right) & u\left(i_{k-1}\right)\end{array}\right]^{T}$. Therefore, from (3) and (4), we can get the following augmented closed-loop system

$$
z(k+1)=\Psi_{k} z(k)
$$

where

$$
\Psi_{k}=\left[\begin{array}{cc}
\Phi_{k}+\Gamma_{0}\left(\tau_{k}, h_{k}\right) K\left(i_{k}\right) & \Gamma_{1}\left(\tau_{k}, h_{k}\right) \\
K\left(i_{k}\right) & 0
\end{array}\right]
$$

It is important to note that $i_{k}$ in (6) refers to the effective sampling instant. Only packets sampled at that instant successfully transmits from sensor to actuator. Since time varying sampling principle is adopted based on (2), $\mathrm{ZOH}$ will be updated only once between two effective sampling instants by control signal. Thus, during any sampling interval, only two control signals will be acted on the plant: $u\left(i_{k-1}\right)$ and $u\left(i_{k}\right)$.

Time delays and packet dropouts are dealt with in a more natural way without any assumptions and limitations. Since the behavior of $\mathrm{ZOH}$ and the time varying sampling principle, the original random delays are transformed to a relative determinate delay which is always less than one 
sampling period. Packet dropouts can be seen as an infinite long time delay.

It can be seen that the newly generated augmented model is a discrete switched linear system with infinite switching rules. Therefore, the problem of stabilizing control for NCS is changed into the stabilization problem of discrete switched linear system.

\section{ASYMPTOTICAL STABILITY ANALYSIS AND CONTROLLER DESIGN}

Based on the selection principle of sampling instants proposed in section II, it can be supposed that as soon as the packet reaches actuator, the next sampling event is driven at sensor. Therefore, $\tau_{k}$ takes value from a finite set $\mathrm{M}=\left\{l, 2 l, \cdots, T_{\max }\right\}$ and the length of interval between two successive effective updating instants $h_{k}$ can be written into:

$$
h_{k}=\tau_{k}+T_{\max } * d_{k}
$$

where $d_{k}$ was defined as the number of dropped packet between two successive effective updating instants.

Thus, (5) can be viewed as a discrete switched system with the particularity that the switching rule defined by $\Psi_{k}$ in (5) which is not known a priori but its instantaneous value is available in real time. However, the total number of switching rules is finite and the value is $N^{*}\left(d_{\max }+1\right)$. Let us define a compact set $I$ where switching rules of (5) lie in, then we can get $I=\left\{1,2,3 \ldots, N^{*}\left(d_{\max }+1\right)\right\}$, Denote $\hat{A}_{i}$ as the switching rule determined by $\tau_{k}$ and $d_{k}$, then, augmented system (11) can be written into a discrete switched system .

$$
z(k+1)=\hat{A}_{i} z(k), \quad \forall i \in I
$$

where

$$
\hat{A}_{i}=\left[\begin{array}{cc}
\Phi_{i}+\Gamma_{i, 0} K_{i} & \Gamma_{i, 1} \\
K_{i} & 0
\end{array}\right]
$$

Considering the switching nature of our system (5), LMI-based quadratic Lyapunov function for asymptotic stability is introduced to check system stability and design stabilizing controller.

Theorem 1: If there exists $N^{*}\left(d_{\max }+1\right)$ symmetric positive definite matrices $S_{1}, S_{2}, \ldots, S_{N^{*}\left(d_{\max }+1\right)}$ and matrices $R_{i}(\forall i \in I)$ satisfying

$$
\left[\begin{array}{cc}
S_{j} & \hat{A}_{i} R_{i} \\
R_{i}^{T} \hat{A}_{i}^{T} & R_{i}+R_{i}^{T}-S_{i}
\end{array}\right]>0, \forall(i, j) \in I
$$

then the system (5) is asymptotically stable.

Proof: If (10) is feasible, then $R_{i}+R_{i}^{T}-S_{j}>0$. It means $R_{i}$ is of full rank. As $S_{i}$ is symmetric positive definite, we have:

$$
\left(S_{i}-R_{i}\right)^{T} S_{i}^{-1}\left(S_{i}-R_{i}\right) \geq 0
$$

which is equivalent to

$$
R_{i}^{T} S_{i}^{-1} R_{i} \geq R_{i}+R_{i}^{T}-S_{i}
$$

$$
\left[\begin{array}{cc}
S_{j} & \hat{A}_{i} R_{i} \\
R_{i}^{T} \hat{A}_{i}^{T} & R_{i}^{T} S_{i}^{-1} R_{i}
\end{array}\right]>0, \forall(i, j) \in I
$$

which is equivalent to:

$$
\left[\begin{array}{cc}
S_{j} & 0 \\
0 & G_{i}^{T}
\end{array}\right]\left[\begin{array}{cc}
S_{j}^{-1} & S_{j}^{-1} A_{i} \\
A_{i}^{T} S_{j}^{-1} & S_{i}^{-1}
\end{array}\right]\left[\begin{array}{cc}
S_{j} & 0 \\
0 & G_{i}
\end{array}\right]>0
$$

Let us denote $P_{i}=S_{i}^{-1}$ and $P_{j}=S_{j}^{-1}$, then (14) is equivalent to

$$
\left[\begin{array}{cc}
P_{j} & \hat{A}_{i}^{T} P_{j} \\
P_{j} \hat{A}_{i} & P_{i}
\end{array}\right]>0, \forall(i, j) \in I
$$

Choose the following form of the Lyapunov function:

$$
V(k)=z^{T}(k) P_{i} z(k)
$$

The difference of Lyapunov function is given by

$$
\begin{aligned}
\Delta V & =V(k+1)-V(k) \\
& =z^{T}(k+1) P_{j} z(k+1)-z^{T}(k) P_{i} z(k) \\
& =z^{T}(k) \hat{A}_{i}^{T} P_{j} \hat{A}_{i} z(k)-z^{T}(k) P_{i} z(k) \\
& =z^{T}(k)\left(\hat{A}_{i}^{T} P_{j} \hat{A}_{i}-P_{i}\right) z(k)
\end{aligned}
$$

According to the Schur complement formula, (15) is equivalent to

$$
P_{i}-\hat{A}_{i}^{T} P_{j} \hat{A}_{i}>0
$$

Thus, if condition (10) holds, then $\Delta V<0$, which implies that system (5) is asymptotically stable.

The following part will study the design of stabilizing controller for NCS.

Let us denote the following matrixes:

$$
\begin{aligned}
& \tilde{A}_{i}=\left[\begin{array}{cc}
\Phi_{i} & \Gamma_{i, 1} \\
0 & 0
\end{array}\right], \tilde{B}_{i}=\left[\begin{array}{c}
\Gamma_{i, 0} \\
I
\end{array}\right], \\
& \tilde{K}_{i}=\left[\begin{array}{ll}
K_{i} & 0
\end{array}\right]
\end{aligned}
$$
form:

Then, system (5) can be rewritten into the following

$$
z(k+1)=\left(\tilde{A}_{i}+\tilde{B}_{i} \tilde{K}_{i}\right) z(k)
$$

Theorem 2: If there exists symmetric positive definite matrices $G_{i}$ and $V_{i}$, matrices $M_{i}, N_{i}, Q_{i}(\forall i \in I)$ satisfying

$$
\left[\begin{array}{cccc}
G_{j} & 0 & \Phi_{i} M_{i}+\Gamma_{i, 0} Q_{i} & \Gamma_{i, 1} N_{i} \\
* & V_{j} & Q_{i} & 0 \\
* & * & M_{i}+M_{i}^{T}-G_{i} & 0 \\
* & * & * & N_{i}+N_{i}^{T}-V_{i}
\end{array}\right]>0, \forall i, j \in I
$$

then the state feedback control given by (3) with

$$
K_{i}=Q_{i} M_{i}^{-1}, \quad i \in I
$$

stabilizes asymptotically the system (5).

Proof: Assume that there exist symmetric positive definite matrices $G_{i}$ and $V_{i}$, matrices matrices $M_{i}, N_{i}, Q_{i}$ ( $\forall i \in I$ ) such that (21) is satisfied. From (22) we get

$$
Q_{i}=K_{i} M_{i}, \quad i \in I
$$

Replacing $Q_{i}$ in (21) by $K_{i} M_{i}$, we can get

Then if (10) holds, then we have 


$$
\left[\begin{array}{cccc}
G_{j} & 0 & \Phi_{i} M_{i}+\Gamma_{i, 0} K_{i} M_{i} & \Gamma_{i, 1} N_{i} \\
* & V_{j} & K_{i} M_{i} & 0 \\
* & * & M_{i}+M_{i}^{T}-G_{i} & 0 \\
* & * & * & N_{i}+N_{i}^{T}-V_{i}
\end{array}\right]>0, \forall i, j \in I
$$

Let us denote

$$
S_{i}=\left[\begin{array}{ll}
G_{i} & \\
& V_{i}
\end{array}\right], R_{i}=\left[\begin{array}{ll}
M_{i} & \\
& N_{i}
\end{array}\right]
$$

And notice that

$$
\left[\begin{array}{cc}
\Phi_{i}+\Gamma_{i, 0} K_{i} & \Gamma_{i, 1} \\
K_{i} & 0
\end{array}\right]=\left[\begin{array}{cc}
\Phi_{i} & \Gamma_{i, 1} \\
0 & 0
\end{array}\right]+\left[\begin{array}{c}
\Gamma_{i, 0} \\
I
\end{array}\right]\left[\begin{array}{ll}
K_{i} & 0
\end{array}\right]=\tilde{A}_{i}+\tilde{B}_{i} \tilde{K}_{i}
$$

Then replacing related item in (24) by (26) (27), we can get

$$
\left[\begin{array}{cc}
S_{j} & \left(\tilde{A}_{i}+\tilde{B}_{i} \tilde{K}_{i}\right) R_{i} \\
R_{i}^{T}\left(\tilde{A}_{i}+\tilde{B}_{i} \tilde{K}_{i}\right)^{T} & R_{i}+R_{i}^{T}-S_{i}
\end{array}\right]>0, \forall(i, j) \in I
$$

Thus, Theorem 1 indicates the sufficient condition for asymptotic stability of closed-loop NCS (5).

\section{EXPONENTIAL STABILITY AND THE BOUND OF DECAY RATE}

Theorem 3: If there exists $N^{*}\left(d_{\max }+1\right)$ symmetric positive definite matrices $S_{1}, S_{2}, \ldots, S_{N^{*}\left(d_{\max }+1\right)}$ and matrices $R_{i}(\forall i \in I)$ satisfying

$$
\left[\begin{array}{cc}
S_{j} & \alpha \hat{A}_{i} R_{i} \\
\alpha R_{i}^{T} \hat{A}_{i}^{T} & R_{i}+R_{i}^{T}-S_{i}
\end{array}\right]>0, \forall(i, j) \in I
$$

then the closed-loop NCS (5) is exponentially stable with a decay rate $\alpha$.

Proof: Choose the following form of the Lyapunov function:

$$
V(k)=z^{T}(k) P_{i} z(k)
$$

Define $\zeta(k)=\alpha^{k} z(k)$ and choose another Lyapunov function:

$$
W(k)=\zeta^{T}(k) P_{i} \zeta(k)
$$

Then the difference for $W(k)$ is given by

$$
\begin{aligned}
\Delta W & =W(k+1)-W(k) \\
& =\zeta^{T}(k+1) P_{j}(k+1) \zeta(k+1)-\zeta^{T}(k) P_{i} \zeta(k) \\
& =\alpha^{2(k+1)} z^{T}(k+1) P_{j}(k+1) z(k+1)-\alpha^{2 k} z^{T}(k) P_{i} z(k) \\
& =\alpha^{2(k+1)} z^{T}(k) \hat{A}_{i}^{T} P_{j} \hat{A}_{i} z(k)-\alpha^{2 k} z^{T}(k) P_{i} z(k) \\
& =\alpha^{2 k} z^{T}(k)\left(\alpha^{2} \hat{A}_{i}^{T} P_{j} \hat{A}_{i}-P_{i}\right) z(k)
\end{aligned}
$$

According to the Schur complement formula and Theorem 1, (28) is equivalent to $P_{i}-\alpha^{2} \hat{A}_{i}^{T} P_{j} \hat{A}_{i}>0$.

Then we can obtain that $\Delta W<0$, which implies $W(k)<W(0)$. Thus we have

$$
V(k)=\alpha^{-2 k} W(k)<\alpha^{-2 k} W(0)=\alpha^{-2 k} V(0)
$$

By the result of theorem 1in [15], we can come to the conclusion that the closed-loop NCS (5) is exponentially stable with a decay rate $\alpha$. This completes the proof.

\section{NUMERICAL EXAMPLE}

In this section, numerical examples will be given to demonstrate the effectiveness of the proposed methods for stabilizing NCS with uncertain time delays and packet dropouts, the bound of decay rate will be found by solving the LMI optimal problem, stability performance with decay rate will be analyzed based on the simulation results. Consider the following plant model:

$$
\begin{aligned}
& \frac{d x}{d t}=\left[\begin{array}{cc}
0 & 1 \\
-2 & -3
\end{array}\right] x+\left[\begin{array}{l}
0 \\
1
\end{array}\right] u \\
& y=\left[\begin{array}{ll}
1 & 0
\end{array}\right] x
\end{aligned}
$$

\section{A. Asymptotical stabilizing control law}

The objective of this example is to obtain asymptotical stabilizing feedback controller gains for the proposed NCS model (5). Suppose the length of gridded equidistant small interval $l$ is $0.05 \mathrm{~ms}$, the values of possible time delays are $\tau_{1}=0.05 \mathrm{~ms}, \tau_{2}=0.1 \mathrm{~ms}, \tau_{3}=0.15 \mathrm{~ms}$; for simplicity, we suppose the bound of consecutive dropped packets $d_{\max }=2$, which means $d_{i}=\{0,1,2\}$. According to permutation and combination, the total number of switching rules is 9 . To obtain feedback gains, we can use the Matlab LMI Control Toolbox to solve the LMI feasible problem presented in (21). The results are as follows. Table 1 shows the combination results of switching rules and the results of controller gains.

Fig. 2 shows the output trajectory of NCS with the feedback control law proposed in this paper. We can see that the networked control system is asymptotical stable. In the next example, we will discuss how the decay rate impacts the stabilizing performance of NCS.

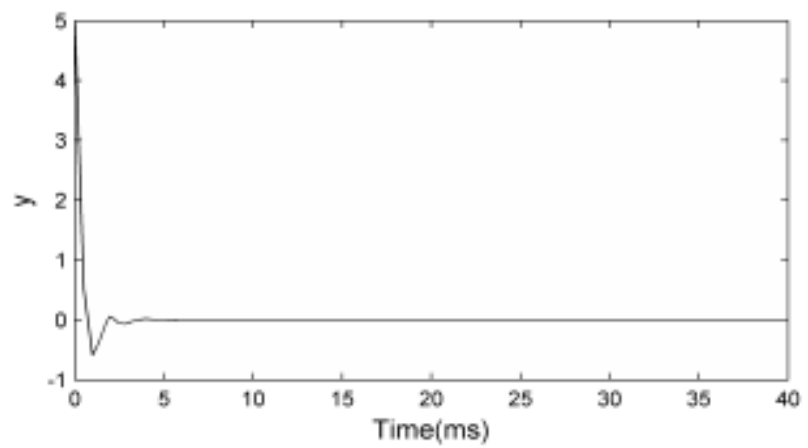

Figure 2. Output trajectory of NCS under asymptotical stabilizing control law

\section{B. Performance analysis with decay rate}

The objective of this example is to obtain the bound of decay rate of the closed-loop NCS (5) and analyze the stabilizing performance of the system with different decay rate. We still use the plant model (32) and apply the same gridding rules with the sample A). By solving the LMI optimal problem with Matlab LMI GEVP solvers, we can get the minimum $\mu=1 / \alpha=0.5042(0<\mu<1)$. Thus, the range of decay rate $\alpha=1 / \mu$ is from 1.0000 to 1.9833 . Fig. 3 shows the stabilizing performance of the system with different decay rate. We can see that decay rate determines the setting time of the output trajectory towards constant value which is the system stable state. The larger the decay 
rate is, the shorter time the system needs to reach the stable state with better stabilizing performance.

Stabilizing control law obtained in example A) can only guarantees NCS to be asymptotically stable, while, The stabilizing performance of control law for NCS can be improved by setting parameter of decay rate in example B.

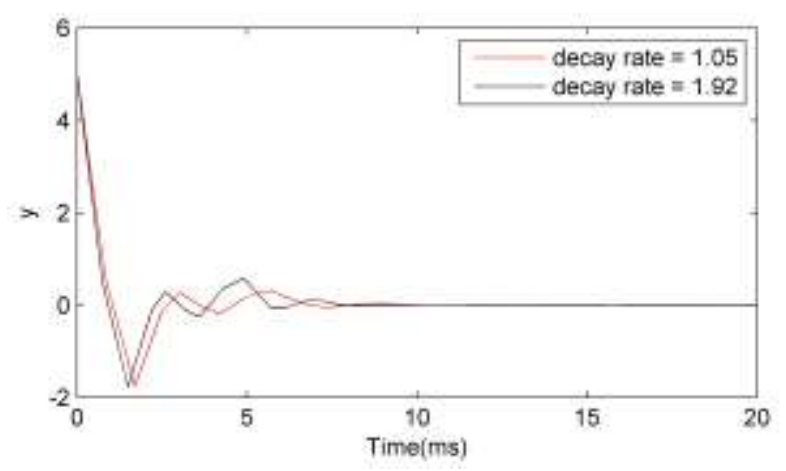

Figure 3. Stabilizing performance of the system with decay rate

\section{CONCLUSIONS}

In this paper, a novel active time varying sampling period strategy is proposed to investigate stability and control design problems with performance analysis for networked control systems.

This method makes sure the time delay between sensor and actuator is always less than one sampling interval, which simplifies the modeling of NCS with uncertain time delays and packet dropouts. An augmented state vector is introduced to successfully convert NCS into a discrete switched linear system. Sufficient conditions for asymptotical stability and exponential stability of proposed NCS model are given. To solve the LMIs for obtaining feedback gains and the bound of decay rate, the "gridding approach" is adopted to guarantee LMI set up for a discrete switched system with finite switching rules. Numerical examples illustrate the effectiveness of the proposed strategy for the asymptotical stabilizing and exponential stabilizing controller over NCS, finally the control performance with decay rate is analyzed based on the simulation results.

\section{REFERENCES}

[1] J.M. Guerrero, J. Matas, L.G. de Vicuna, M. Castilla, J. Miret, "Wireless-control strategy for parallel operation of distributedgeneration inverters," IEEE Trans. Ind. Electron., vol.53, 2006, pp.1461-1470.

[2] H.S. Park, Y.H. Kim, D.S. Kim, W.H. Kwon, "A scheduling method for network-based control systems," IEEE Trans. Control Syst. Technol., vol.10, 2002, pp.318-330.

[3] W. Zhang, M.S. Branicky, S.M. Phillips, "Stability of networked control systems," IEEE Control Syst. Mag., vol.21, 2001, pp.84-99.

[4] Y. Tipsuwan, M.Y. Chow, "Control methodologies in networked control systems," Control Eng. Pract., vol.11, 2003, pp.1099-1111.

[5] G.C. Walsh, H. Ye, L.G. Bushnell, "Stability analysis of networked control systems," IEEE Trans. Control Syst. Technol., vol.10, 2002, pp.438-446.

[6] J.1. Meng, Y.y. Yang, C.j. Yang, L. Yang, "Modeling, Qualitative Analysis and Design of Networked Control system," International Conference of China Communication (Iccc2010), 2010, pp.797802.

[7] R. Luck, A. Ray, "Experimental-Verification of a Delay Compensation Algorithm for Integrated Communication and Control Systems" Int. J. Control, vol.59, 1994, pp.1357-1372.

[8] H.B. Li, M.Y. Chow, Z.Q. Sun, "Optimal Stabilizing Gain Selection for Networked Control Systems With Time Delays and Packet Losses," IEEE Trans. Control Syst. Technol., vol.17, 2009, pp.1154-1162.

[9] L.A. Montestruque, P.J. Antsaklis, "On the model-based control of networked systems," Automatica, vol.39, 2003, pp.1837-1843.

[10] B. Yu, Y. Shi, Y. Lin, "Discrete-time H-2 output tracking control of wireless networked control systems with Markov communication models," Wirel. Commun. Mob. Comput., vol.11 2011, pp.1107-1116.

[11] A. Sala, "Computer control under time-varying sampling period: An LMI gridding approach," Automatica, vol.41, vol.2005, pp.2077-2082.

[12] V.T. Minh, M. Awang, S. Parman, "Conditions for Stabilizability of Linear Switched Systems," International Journal of Control Automation and Systems, vol.9, 2011, pp.139-144.

[13] W. Zhang, A. Abate, J.H. Hu, M.P. Vitus, "Exponential stabilization of discrete-time switched linear systems," Automatica, vol.45, 2009, pp.2526-2536.

[14] L. Zhang, P. Shi, M. Basin, "Robust stability and stabilisation of uncertain switched linear discrete time-delay systems," IET Control Theory Appl., vol.2, 2008, pp.606-614.

[15] W.A. Zhang, L. Yu, "Output feedback stabilization of networked control systems with packet dropouts," IEEE Trans. Autom. Control, vol.52, 2007, pp.1705-1710.

TABLE I. COMBINATION RESULTS OF SWITCHING RULES AND RESULTS

\begin{tabular}{cccc}
\hline & $\tau_{1}=0.05 m s$ & $\tau_{2}=0.1 \mathrm{~ms}$ & $\tau_{3}=0.15 m s$ \\
\hline$d_{1}=0$ & $K_{1}=\left[\begin{array}{lll}-4.5191 & -4.7528\end{array}\right]$ & $K_{2}=\left[\begin{array}{lll}-2.9523 & -3.1847\end{array}\right]$ & $K_{3}=\left[\begin{array}{lll}-1.6277 & -1.7985\end{array}\right]$ \\
$d_{2}=1$ & $K_{4}=\left[\begin{array}{lll}-2.0723 & -2.3737\end{array}\right]$ & $K_{5}=\left[\begin{array}{lll}-1.6946 & -2.0218\end{array}\right]$ & $K_{6}=\left[\begin{array}{ll}-1.2932 & -1.5992\end{array}\right]$ \\
$d_{3}=2$ & $K_{7}=\left[\begin{array}{lll}-1.4100 & -1.8404\end{array}\right]$ & $K_{8}=\left[\begin{array}{lll}-0.7153 & -0.9711\end{array}\right]$ & $K_{9}=\left[\begin{array}{ll}-1.5746 & -2.3864\end{array}\right]$ \\
\hline
\end{tabular}

